\title{
A relação entre a habilidade de leitura e a consciência fonológica: estudo longitudinal em crianças pré- escolares
}

\section{The relationship between reading skills and phonological awareness: a Longitudinal study in preschool children}

\author{
Antonio Roazzi* \\ Universidade Federal de Pernambuco - UFPE, Recife, Pernambuco, Brasil
}

Maira M. Roazzi**

Universidade Federal do Rio Grande do Sul, Porto Alegre, Rio Grande do Sul, Brasil

Cláudia Nascimento Guaraldo J usti***

Universidade Federal de Juiz de Fora, Juiz de Fora, Minas Gerais - Brasil

Francis Ricardo dos Reis J usti***

Universidade Federal de Juiz de Fora, Juiz de Fora, Minas Gerais - Brasil

\begin{abstract}
RESUMO
O trabalho apresenta os resultados de uma pesquisa longitudinal em crianças falantes de língua portuguesa, investigando a relação entre consciência fonológica e a aprendizagem da leitura. A consciência fonológica foi medida por meio de tarefas que envolviam a fusão e a segmentação silábica e fonêmica que foram aplicadas na pré-escola e no final do primeiro ano de alfabetização. Análises de regressão linear múltipla controlando a idade e a inteligência (verbal e não verbal) foram realizadas e apenas os escores na tarefa de fusão silábica aplicada no final do primeiro ano de alfabetização predisseram os escores em um teste de leitura aplicado no mesmo período. Porém análises não paramétricas indicaram que ambas as tarefas de fusão (fonêmica e silábica) aplicadas na pré-escola predisseram a leitura um ano depois. Esses resultados foram discutidos considerando-se a natureza das tarefas de consciência fonológica empregadas e o seu papel em relação ao aprendizado da leitura.
\end{abstract}

Palavras-chave: Consciência fonológica, Leitura, Fusão e segmentação fonêmica/silábica.

\section{ABSTRACT}

This paper presents the results of a longitudinal research on portuguesespeaking children, investigating the relationship between phonological awareness and reading acquisition. Phonological awareness was accessed on two periods of time: on the pre-school and on the end of the first year of

\begin{tabular}{|l|l|l|l|l|l|}
\hline Estudos e Pesquisas em Psicologia & Rio de Janeiro & v. 13 & n. 2 & p. 420-446 & 2013 \\
\hline
\end{tabular}


Antonio Roazzi, Maira M. Roazzi, Cláudia Nascimento Guaraldo Justi, Francis Ricardo dos Reis J usti A relação entre a habilidade de leitura e a consciência fonológica: estudo longitudinal em crianças pré-escolares

reading education. Phonological awareness was indexed by tasks involving fusion (phonemic and syllabic) and segmentation (phonemic and syllabic). Multiple regression analyses controlling for age and intelligence (verbal and non-verbal) indicated that only the syllabic fusion task accessed on the first year of education predicted reading. However non-parametric analyses revealed that both phonemic and syllabic fusion tasks accessed on pre-school predicted reading one year later. The nature of phonological awareness tasks and its relations with reading acquisition were discussed.

Keywords: Phonological awareness, Reading, Merger and phonemic segmentation / syllabic

\section{I ntrodução}

Nas últimas décadas, tem sido observado um interesse crescente de vários pesquisadores em estabelecer os elos de ligação e as possíveis conexões causais existentes entre a emergência de uma tomada de consciência da linguagem como objeto de reflexão, ou seja, de uma capacidade para refletir sobre os diferentes aspectos estruturais da própria linguagem, conhecida como consciência metalinguística, e o desenvolvimento da leitura e da escrita (BRADLEY; BRYANT, 1983। 1991; BRYANT; BRADLEY, 1985; CARDOSO-MARTINS, 1995a; CASTLES; COLTHEART, 2004; DILLON; JONG; PISONI, 2011; INVERIZZI, 2003; JORM; SHARE, 1983; MOTA, 2009; PHILLIPS; TORGESEN, 2006; SHARE; JORM; MC LEAN; MATTHEWS, 1984; OLOFSSON LUNDBERG, 1985; ROAZZI, 1992; ROAZZI, DOWKER; BRYANT, 1993; ROAZZI; ASFORA; QUEIROGA; DIAS, 2010; SNOWLING, 1987; WAGNER; TORGESEN, 1987).

A consciência metalinguística engloba diferentes habilidades, como, por exemplo, a consciência fonológica (habilidade de refletir sobre e manipular os sons que compõem a fala), a consciência morfológica (habilidade de refletir sobre e manipular as menores unidades da fala que possuem significado, ou seja, os morfemas), a consciência sintática (habilidade de refletir sobre e manipular a sintaxe da língua, ou seja, a ordenação das palavras nas sentenças) e a consciência metatextual (habilidade de refletir sobre a estrutura do texto, suas partes, suas convenções linguísticas, etc.). Dessas quatro habilidades metalinguísticas, a mais amplamente investigada tem sido a consciência fonológica (MOTA, 2009).

O interesse inicial no estudo da relação entre a consciência fonológica e o desenvolvimento da leitura surgiu a partir da constatação referente à estrutura de nosso sistema de escrita, o alfabético, que implica na necessidade de se estabelecer uma conexão entre os sons da fala e a linguagem escrita. De fato, para adquirir a correspondência grafo- 
Antonio Roazzi, Maira M. Roazzi, Cláudia Nascimento Guaraldo Justi, Francis Ricardo dos Reis J usti A relação entre a habilidade de leitura e a consciência fonológica: estudo longitudinal em crianças pré-escolares

fonêmica, princípio específico do sistema alfabético, o aprendiz leitor deve possuir um mínimo de consciência fonológica: a consciência da decomposição das palavras em elementos de diferentes dimensões (sílabas, fonemas) desprovidos de qualquer significado e com possibilidade de serem combinados entre si.

\section{Qual a direção da relação causal entre a consciência fonológica e a leitura?}

A relação existente entre a consciência fonológica e o desenvolvimento da leitura e da escrita pode ser interpretada pelo menos de três formas: causa (a consciência fonológica desempenha um papel causal na aquisição da leitura e da escrita); consequência (a consciência fonológica é consequência do aprendizado da leitura e da escrita); e, causação recíproca (a consciência fonológica afeta o desenvolvimento da leitura e da escrita, e este, por sua vez, afeta o desenvolvimento da consciência fonológica).

De acordo com Roazzi (1993), duas versões podem ser identificadas dentro da posição que concebe a consciência fonológica como desempenhando um papel causal na aquisição da leitura e da escrita: uma que considera a relação causal de uma maneira mais rígida e a outra que considera a relação causal de uma maneira mais flexível. Para os defensores da versão mais rígida, uma boa consciência fonológica seria um pré-requisito indispensável para a aquisição da leitura e da escrita. Já os defensores da versão mais flexível, veem a consciência fonológica como uma habilidade precursora da aquisição da leitura e da escrita, e não como um pré-requisito.

A proposta de Bryant e Bradley (1985), por exemplo, não é de que a consciência das unidades fonológicas faz com que a criança seja capaz de ler, mas que essa consciência permite um melhor aprendizado da leitura algum tempo mais tarde. Esses pesquisadores argumentaram que a consciência de unidades maiores da fala, tais como as rimas (a palavra / paR/ rima com a palavra /maR/, assim como / doR/ com / koR/), poderia auxiliar a criança a mapear esses sons, em sequências de letras, tais como 'ar' e 'or'. Goswami e Bryant, (1990) ressaltam que as crianças, através de analogias, logo compreenderiam que palavras que rimam compartilham letras em comum e utilizariam este conhecimento para ler palavras desconhecidas que compartilham a mesma sequência final de letras das palavras conhecidas. Para esses pesquisadores, quando uma pessoa se dá conta de que duas palavras rimam, por exemplo, ela está dissecando os sons dessas palavras. No momento que se sabe que /paR/ e / $\mathrm{maR} /$ têm um som em comum e que este som é 
/aR/, decompõe-se cada uma dessas duas palavras em menores unidades de som. Assim sendo, a criança que compreende a rima, de acordo com Goswami e Bryant (1990), deve saber algo sobre os sons constituintes das palavras. Bryant e Bradley (1985) salientam que a rima é a base dos jogos de palavras e das cantigas de roda, sendo, portanto, parte marcante do começo da vida das crianças. Segundo esses autores, se as crianças detectam as rimas, devem, de alguma maneira, ser também conscientes dos sons da fala e, uma vez que se tratam de crianças muito pequenas - que ainda estão longe de começar a ler - está implícito que esse tipo de consciência precede a leitura por um período de tempo significativo.

Para avaliar a hipótese de que a consciência fonológica é precursora da aquisição da leitura, Bryant e Bradley (1985) realizaram um estudo longitudinal na Inglaterra com 400 crianças pré-escolares. No início do estudo, nenhuma das crianças havia ainda começado a ler. A avaliação da consciência fonológica consistia em ministrar séries de tarefas de detecção de rima e aliteração. Nessas tarefas, a criança escutava três ou quatro palavras, duas ou três das quais tinham um som em comum. A criança tinha que ouvir as palavras e indicar aos pesquisadores qual das palavras destoava das demais. A posição do som na palavra alvo variava. Em alguns itens estava no final (por exemplo: 'pin', 'win', 'sit'), em outros, no meio (por exemplo: 'pot', 'cat', 'hat') e em alguns, no início (por exemplo: 'pig', 'hill', 'pin'). Nos dois primeiros casos, as crianças tinham que indicar quais palavras rimavam; no terceiro, tinham que se dar conta da aliteração (som inicial que se destoava). Ao atingirem a idade de oito ou nove anos, as crianças foram novamente submetidas à avaliação do nível de desenvolvimento da leitura. Os resultados das análises revelaram que os escores das crianças nos testes iniciais de rima e aliteração predisseram o progresso na leitura e na escrita três ou quatro anos mais tarde. A relação se manteve constante mesmo após os autores terem controlado os efeitos de variações na inteligência, na memória de trabalho fonológica e na educação materna.

É interessante considerar que se por um lado, os resultados de diversos estudos longitudinais dão suporte à tese de que a consciência fonológica é precursora do desenvolvimento da leitura e da escrita (BRYANT; BRADLEY, 1987; BRYANT; MACLEAN; BRADLEY; CROSSLAND, 1990; CARDOSO-MARTINS, 1991; PESTUN, 2005; WIMMER LANDERL LINORTNER; HUMMER, 1991), por outro lado, essa tese também recebe suporte de estudos que estimularam a consciência fonológica. Vários desses estudos evidenciaram um efeito positivo da estimulação da consciência fonológica sobre a própria consciência fonológica e sobre o desenvolvimento da leitura e da escrita (BALL; BLACHMAN, 1991; 
BRYANT; BRADLEY, 1985; BRADY; FOWLER; STONE; WINBURY, 1994; BYRNE; FIELDING-BARNSLEY, 1995; CAPOVILLA; CAPOVILLA, 2000; CÁRNIO; SANTOS, 2005; FUKUDA; CAPELLINI, 2012; HEIN; TEIXEIRA; SEABRA; MACEDO, 2010; BERNARDINO JUNIOR; FREITAS; SOUZA; MARANHE; BANDINI, 2006; JUSTINO; BARREIRA, 2012; PAULA; MOTA; KESKE-SOARES, 2005). Por exemplo, Capovilla e Capovilla (2000) desenvolveram um estudo que envolveu o treinamento da consciência fonológica em crianças brasileiras que apresentavam dificuldade nessa habilidade. O estudo contou com um grupo experimental e com dois grupos controle: o grupo experimental era constituído por crianças com baixo desempenho em tarefas de consciência fonológica, um dos grupos controle também era constituído por crianças com baixo desempenho em tarefas de consciência fonológica e o outro grupo controle era constituído por crianças com um bom desempenho nessas tarefas. Os pesquisadores observaram que, após o treinamento da consciência fonológica, o grupo experimental superou em desempenho o grupo controle que também tinha dificuldades na consciência fonológica e se igualou em desempenho ao grupo controle que apresentava bom desempenho nas habilidades fonológicas. Isso aconteceu tanto para as habilidades fonológicas quanto para as habilidades de leitura e de escrita. Esse resultado indica que o treino da consciência fonológica não foi apenas positivo, mas foi também forte o suficiente para igualar os participantes que tinham um desempenho ruim nessa atividade à participantes que tinham um bom desempenho. Em especial, é importante ressaltar que, corroborando o papel causal da consciência fonológica para o desenvolvimento da leitura e da escrita, o grupo experimental apresentou ganhos também nessas últimas habilidades, mesmo tendo sido treinados apenas nas habilidades de consciência fonológica.

Apesar das evidências em favor da interpretação de uma relação de causalidade entre a consciência fonológica e a leitura e a escrita, no sentido da consciência fonológica facilitar a aquisição da leitura e da escrita, essa interpretação foi questionada por alguns pesquisadores que defendem que é o processo de aprender a ler e a escrever, por si mesmo, que alerta o aprendiz para a relevância dos segmentos fonológicos na linguagem. Por exemplo, Morais (1996), avaliaram a consciência fonológica de adultos portugueses iletrados, que jamais tinham aprendido a ler e a escrever, comparando-os a ex-iletrados, isso é, pessoas que tinham aprendido a ler e a escrever na idade adulta, em classes de alfabetização. Os participantes foram submetidos a tarefas de adição e subtração de fonemas e de sílabas. Segundo o pesquisador, os adultos iletrados foram incapazes de realizar as tarefas que envolviam a adição e a subtração de fonemas (não se tratava de uma dificuldade de 
compreender a operação de subtração, pois a maioria dos iletrados conseguira êxito nas tarefas de subtração e adição de sílabas). Em compensação, a grande maioria dos ex-iletrados conseguiu realizar a tarefa de subtração de fonemas. Um estudo realizado com adultos falantes do português brasileiro que também corrobora os achados de Morais (1996) é o de Mota e Castro (2007). Nesse estudo, Mota e Castro compararam o desempenho de adultos iletrados com adultos alfabetizados em tarefas de detecção de rima e de aliteração e observaram que os adultos iletrados tiveram um desempenho pior em ambas as tarefas. Desse modo, os achados de Mota e Castro, ampliam os achados de Morais (1996) para unidades fonológicas mais amplas que o fonema e a sílaba. Porém é necessária certa precaução na interpretação dos resultados de Mota e Castro porque o grupo de adultos iletrados contou com apenas cinco participantes e esse número tão reduzido aumenta as chances dos resultados se deverem a idiossincrasias desses indivíduos.

Apesar da aparente inconsistência nos resultados dos estudos no que se refere à relação entre a consciência fonológica e a leitura e a escrita, essa inconsistência desaparece ao se considerar que a consciência fonológica não se caracteriza por ser uma “... variável única, binária, dicotômica" (GOUGH; LARSON; YOPP, 1995, p. 15), algo que alguém tenha ou não, pois se refere à consciência de diferentes unidades fonológicas (ROAZZI; DOWKER, 1989). O termo 'consciência fonológica' refere-se à consciência dos sons da fala e inclui não somente a consciência dos fonemas, mas também, a consciência de unidades fonológicas maiores (palavras, sílabas, rimas). Dessa forma, é possível que enquanto à consciência de algumas unidades fonológicas se desenvolva antes do ensino formal da leitura e seja precursora da aquisição da leitura, a consciência de outras unidades fonológicas só se desenvolva a partir do aprendizado da leitura e da escrita. Por exemplo, no próprio estudo relatado por Morais (1996), os portugueses iletrados não tiveram êxito no desempenho em tarefas de consciência fonêmica, mas tiveram êxito no desempenho em tarefas de consciência silábica.

Perfetti, Beck, Bell, e Hughes (1987) apresentam evidências que vão ao encontro de uma perspectiva interativa da relação entre o desenvolvimento da consciência fonológica e o desenvolvimento da leitura. Perfetti et al. desenvolveram um estudo longitudinal no qual 82 crianças que cursavam a primeira série do ensino fundamental foram avaliadas em suas habilidades de leitura e de consciência fonológica. Os resultados encontrados evidenciaram que, enquanto, por um lado, a consciência fonológica facilitou a aprendizagem da leitura, por outro lado, o progresso na leitura promoveu o desenvolvimento da consciência fonológica, avaliada através de tarefas de segmentação de palavras. 0 
mesmo tipo de relação bidirecional foi encontrado por Roazzi e Carvalho (1995) em 120 crianças brasileiras com idades entre cinco e sete anos.

Em suma, de uma forma geral, os resultados de diversos estudos indicam que enquanto a consciência fonêmica se desenvolveria como uma consequência do aprendizado da leitura e da escrita, a consciência de unidades fonológicas maiores que o fonema poderia estar presente antes do aprendizado da leitura e da escrita e, nesse caso, favoreceria a aprendizagem da leitura e da escrita. Dessa forma, a relação entre a consciência fonológica e a aquisição da leitura seria recíproca, com aumentos em uma servindo de suporte para o desenvolvimento da outra. Essa forma de interpretar a relação existente entre a consciência fonológica e a leitura parece ser a mais aceita pelos pesquisadores (BARRERA, 2003; BOWEY, 1994; BURGESS; LONIGAN, 1998; CARDOSO-MARTINS, 1991; CASTLES; HOLMES; NEATH; KINOSHITA, 2003; MALUF; BARRERA, 1997; MANRIQUE; SIGNORINI, 1988; MORAIS; ALEGRIA; CONTENT, 1987; PESTUN, 2005; WAGNER; TORGESEN; RASHOTTE, 1994).

\section{A relação entre as diferentes medidas de consciência fonológica e o desenvolvimento da leitura e da escrita}

Uma breve pesquisa na literatura da área evidencia a variabilidade das tarefas utilizadas pelos pesquisadores para mensurar a consciência fonológica. Por exemplo, as medidas utilizadas podem requerer que o indivíduo: 1) faça a fusão de vários fonemas ou sílabas ouvidas e diga a palavra correspondente; 2) diga qual palavra resulta da inserção ou da subtração de um fonema no começo de uma palavra ouvida; 3) bata sobre a mesa de acordo com o número de fonemas ou sílabas presentes em uma palavra ouvida; 4) inverta as sílabas de palavras dissílabas, etc. Estas várias tarefas medem aspectos diferentes da consciência fonológica e podem se desenvolver no indivíduo de forma diferente. Por exemplo, existem evidências na literatura de que habilidades de segmentação explícita (e.g., subtração da primeira consoante) não emergem de forma espontânea, enquanto a análise das palavras em sílabas e a detecção de similaridades fonológicas (e.g., a habilidade de detectar rimas) podem emergir espontaneamente (e.g., BERTELSON; DE GELDER, 1991; DE GELDER, 1990; DE GELDER; VROOMEN, 1991). Estas últimas habilidades parecem depender muito menos do nível de alfabetização possuído pelo sujeito, dado que não dependem diretamente de uma representação segmentada da palavra, dependem mais de uma habilidade intuitiva de perceber similaridades fonológicas globais (CARDOSO-MARTINS, 1994), enquanto as primeiras podem ser 
adquiridas depois do sujeito ser submetido a treinamentos explícitos, como também depois do sujeito ter aprendido a dominar a língua escrita.

Como a consciência fonológica vem sendo medida através do uso de diferentes tipos de tarefas, que se diferenciam tanto por suas demandas cognitivas (identificação, fusão, segmentação) quanto pela unidade de som envolvida (rima, sílaba, fonema), é possível que as habilidades subjacentes à realização destas tarefas sejam qualitativamente diferentes entre si (VLOEDGRAVEN; VERHOEVEN, 2009). Apesar de ser logicamente possível conceber que as diferentes tarefas de consciência fonológica representam um construto multidimensional, existem evidências de que as diferentes tarefas utilizadas para avaliar a consciência fonológica indexam, de fato, um construto unidimensional (SCHATSCHNEIDER; FRANCIS; FOORMAN; FLETCHER, 1999; STANOVICH; CUNNIGHAM; CRAMER, 1984; TRESSOLDI, 1989; VLOEDGRAVEN; VERHOEVEN, 2009). Entretanto, isso não significa que essas diferentes tarefas não variem em sua capacidade de prover informação sobre a habilidade de consciência fonológica dos indivíduos. Existem evidências que revelam uma relação diferente entre o desenvolvimento da leitura e da escrita e algumas destas tarefas de consciência fonológica como, por exemplo, as tarefas que envolvem a segmentação e a fusão fonêmica. Por exemplo, Perin (1983) comparou dois grupos de sujeitos em uma tarefa de segmentação fonêmica, sendo um grupo com nível de desempenho baixo e outro com nível de desempenho alto na habilidade de escrita de palavras (tradução da palavra 'spelling' em inglês). Os resultados mostraram um nível de segmentação fonêmica inferior no grupo com baixo nível de desempenho na tarefa de escrita, em relação ao grupo com alto nível de desempenho, mas com deficiência na leitura. Estes dados sugerem que as habilidades de segmentação fonêmica estão mais ligadas ao desenvolvimento da escrita do que da leitura.

Os resultados de Fox e Routh (1984) são compatíveis com a ideia de que a fusão fonêmica parece ser mais importante para a leitura do que a segmentação fonêmica. Fox e Routh compararam a leitura de dois grupos de crianças submetidas a diferentes tipos de treinamento. $O$ primeiro grupo foi treinado em tarefas de fusão e segmentação fonêmica, enquanto o segundo grupo foi treinado exclusivamente em tarefas de segmentação fonêmica. Encontrou-se que o primeiro grupo aprendeu a ler melhor do que o segundo grupo, indicando, desta forma, que a habilidade de fusão fonêmica desempenha um papel mais pronunciado na aprendizagem da leitura do que a segmentação fonêmica. 
No entanto, em um estudo longitudinal, Lundberg e Hoien (1991), diferentemente de Perin (1983), encontraram que não só as habilidades de escrita de crianças de terceira série, mas também as habilidades de leitura podiam ser previstas a partir do nível de desempenho em uma tarefa de segmentação fonêmica destas mesmas crianças quando cursavam a pré-escola. Além disso, os resultados do estudo de Cossu, Shankweiler, Liberman, Tola, e Katz (1988) vão na mesma direção dos resultados de Lundberg e Hoien, no que se refere à importância da segmentação fonêmica para o desenvolvimento da leitura. Os pesquisadores realizaram um estudo comparando crianças italianas e americanas em habilidades de segmentação fonológica e de leitura. Os resultados evidenciaram uma diferença significativa entre bons e maus leitores, independentemente de serem italianos ou americanos, na segmentação de palavras em fonemas, no sentido de que os piores leitores também eram os que tinham mais dificuldade na tarefa de segmentação fonêmica.

\section{A razão do estudo}

A partir das evidências acima apresentadas, torna-se claro que o conhecimento sobre o papel da consciência fonológica no desenvolvimento da leitura e da escrita não está compreendido em seus detalhes. Por exemplo, os estudos que investigaram a relação entre, por um lado, a segmentação e a fusão fonêmica e, por outro lado, o desenvolvimento da leitura e da escrita têm apresentado resultados inconsistentes. Uma possível explicação para tal inconsistência é a falta de controle das habilidades intelectuais, sendo possível, portanto, que os resultados reflitam variações nos níveis de desenvolvimento intelectual das crianças. Outro ponto negativo dos estudos é que eles se limitaram a realizar análises de correlação, sendo que a utilização de regressões múltiplas permitiria um maior controle de outras variáveis que poderiam estar afetando o desempenho dos sujeitos. Assim sendo, o presente estudo tem como objetivo verificar, longitudinalmente, a contribuição específica da segmentação e da fusão fonêmica e silábica para o desenvolvimento da leitura, controlando a influência de variações na inteligência verbal e não verbal.

O fato do presente estudo ser longitudinal e de envolver crianças brasileiras em idade pré-escolar merece destaque, tendo em vista que, dos 23 estudos recuperados na base de dados 'SCIELO', em uma pesquisa realizada em maio de 2013 (descritor: consciência fonológica), apenas um se trata de um estudo longitudinal que incluiu crianças brasileiras na fase pré-escolar (PESTUN, 2005). O estudo de Pestun teve 
como objetivo verificar a relação entre a consciência fonológica e o desenvolvimento da leitura e da escrita. As crianças foram avaliadas no início do último ano da pré-escola quanto à consciência fonológica e reavaliadas quando se encontravam no início e no término do 10 ano. Tarefas que avaliam as habilidades de leitura e de escrita foram aplicadas quando as crianças estavam no início e no término do 10 ano. Pestun dividiu as crianças em dois grupos de acordo com o escore em consciência fonológica nos três momentos: crianças que ficaram acima da média no teste de consciência fonológica e crianças que ficaram abaixo da média. Os escores desses dois grupos de crianças nas medidas de leitura e de escrita no início e no final do primeiro ano escolar foram comparados e as crianças classificadas como acima da média em consciência fonológica tiveram um desempenho significativamente superior em leitura e em escrita do que as crianças classificadas como abaixo da média em consciência fonológica. No entanto, infelizmente, pode-se dizer que as habilidades de consciência fonológica no estudo de Pestun foram consideradas apenas de forma global, posto que, apesar das crianças terem sido avaliadas quanto às habilidades de segmentação silábica e fonêmica e fusão silábica e fonêmica, esses escores foram somados para representar a habilidade de consciência fonológica na análise dos dados. Assim sendo, embora traga evidências sobre a direção da relação entre a consciência fonológica e a leitura em falantes do português brasileiro, o estudo de Pestun não ilumina o papel das habilidades de segmentar e aglutinar unidades fonológicas nessa relação.

Outro estudo digno de nota que foi realizado com crianças pré-escolares falantes do português brasileiro é o já clássico estudo de CardosoMartins (1995b). Nesse estudo Cardoso-Martins investigou, longitudinalmente, a contribuição de habilidades de consciência fonológica, medidas na pré-escola, para as habilidades de leitura e de escrita, medidas no final do 1 o ano escolar. As habilidades de consciência fonológica investigadas foram a detecção de rimas, a detecção de sílabas, a deteç̧ão de fonemas e a segmentação de fonemas. A pesquisadora realizou análises de regressão linear múltipla controlando a inteligência e as habilidades iniciais de leitura e escrita e observou que o desempenho nas tarefas de detecção de sílaba e de segmentação fonêmica, medidas na pré-escola, contribuíram de forma significativa e independente para as habilidades de leitura e de escrita, medidas no final do primeiro ano escolar. Assim sendo, embora o estudo de Cardoso-Martins (1995b) tenha analisado, especificamente, a contribuição de diferentes níveis da consciência fonológica para a leitura, o estudo não utilizou medidas que envolvessem fusão de segmentos fonológicos, impossibilitando, assim, avaliar se as tarefas que requerem 
fusão são diferentes das que requerem segmentação no que diz respeito à sua relação com a leitura.

Em suma, considerando-se a pesquisa realizada na base de dados 'SCIELO' e os estudos de Pestun (2005) e de Cardoso-Martins (1995b), percebe-se que há uma carência de pesquisas no cenário nacional que objetivaram investigar longitudinalmente as contribuições diferenciais das habilidades de segmentação e de fusão de unidades fonológicas, medidas na pré-escola, para a habilidade de leitura futura. A inclusão de medidas de segmentação e fusão de fonemas e de sílabas possibilita analisar tanto a hipótese de que as tarefas que envolvem fusão são mais importantes para a leitura do que as tarefas que envolvem segmentação quanto a questão de que o nível de desenvolvimento da leitura da criança afeta o poder preditivo das tarefas de consciência fonológica (a consciência fonêmica só se desenvolveria depois do aprendizado da leitura, enquanto a silábica se desenvolveria antes). O fato das crianças ainda não estarem aprendendo a ler (pré-escola) e o fato do estudo ser longitudinal propicia inferências sobre a direção da causalidade entre a consciência fonológica e a leitura. Assim sendo, o presente estudo se justifica não só porque existem inconsistências nos resultados dos estudos que investigaram a relação entre a segmentação e a fusão fonêmica e o desenvolvimento da leitura, mas também porque se trata de um estudo longitudinal que tem início antes das crianças serem ensinadas a ler e que por incluir medidas de segmentação e fusão de fonemas e de sílabas e analisar o papel de cada uma no desenvolvimento da leitura tem o potencial de lançar luz sobre pontos ainda não esclarecidos por estudos anteriores.

\section{Método}

\subsection{Participantes}

Participaram da pesquisa 69 crianças de uma escola da rede particular que frequentavam o último período da pré-alfabetização.

\subsection{Instrumentos}

Inteligência Verbal e Não Verbal

- Subteste vocabulário do WISC (Wechsler, 1991/2002) - Usado como uma medida de inteligência verbal.

- Matrizes Progressivas de Raven - forma especial (Raven, 1987) Usado como uma medida de inteligência verbal.

Consciência fonológica 
- Tarefa de fusão e segmentação fonêmica: A primeira parte desta tarefa requer que a criança aglutine os fonemas enunciados pelo experimentador e diga a palavra ou a pseudopalavra resultante. A tarefa foi introduzida da seguinte forma:

"Eu estou interessado no que as crianças sabem; estou interessado sobre o que as crianças pensam sobre as palavras. Então, agora vamos brincar de E.T.

Este boneco é um E.T. Ele fala de um jeito diferente. Eu gravei algumas palavras que ele falou para que você aprenda a falar a língua dele (a criança escutava os seguintes fonemas no gravador: /s/, /a/, $/ \mathrm{p} /, / \mathrm{U} /$ ). Você descobriu o que ele falou?"

Se a criança não descobrisse, o experimentador dizia qual era a palavra. Caso a criança não compreendesse a instrução, o experimentador fazia novas demonstrações até que ela entendesse e só assim começava o teste. As palavras e as pseudopalavras utilizadas podem ser visualizadas na tabela 1.

A segunda parte dessa tarefa requer que a criança segmente os estímulos (que também podiam ser palavras ou pseudopalavras) enunciados pelo experimentador, pronunciando os fonemas presentes nos mesmos, separadamente. A criança era instruída a falar do mesmo modo que o E.T., para que o E.T. conseguisse entendê-la. Os estímulos usados encontram-se na Tabela 1.

Tabela 1. Lista de palavras utilizadas na tarefa de segmentação e E fusão fonológica

\begin{tabular}{ccccccccc}
\hline \multicolumn{3}{c|}{ Palavras } & \multicolumn{4}{c}{ Não-palavras } \\
\multicolumn{2}{c}{ Simples } & \multicolumn{2}{c}{ Complexas } & \multicolumn{2}{c}{ Simples } & Complexas \\
\hline Dissílib & Trissílib & Dissílib & Trissílib & Dissílib & Trissílib & Dissílib & Trissílib \\
\hline $\begin{array}{c}\text { Grupo A } \\
\text { casa }\end{array}$ boneca & prato & martelo & fuco & salemu & pleta & asdoma \\
bola & sapato & livro & caderno & leco & futovi & bleto & vierne \\
\hline $\begin{array}{c}\text { Grupo B } \\
\text { vela }\end{array}$ farofa & blusa fósforo & tufi & tivabé & vroti & cafoste \\
pato & favela & clube & pistola & vico & polomo & brifo & espito \\
\hline Dissílabas: dissílabas; Trissílib: trissílabas & & & &
\end{tabular}

É importante mencionar que o grupo de crianças que fez a parte de fusão com o grupo A de estímulos descrito acima, fazia a tarefa de segmentação com o grupo B e vice-versa, para não se correr o risco de treinamento. 
- Tarefa de fusão e segmentação silábica: para avaliar a fusão e a segmentação silábica foi utilizada uma brincadeira tradicional no Brasil ("Língua do Pê"), na qual as crianças dividem as palavras em sílabas e acrescentam o "Pê" no início de cada sílaba, formando, assim, um código para a comunicação de mensagens "secretas". Foram apresentadas duas listas: uma com palavras e outra com pseudopalavras. A tarefa é dividida em duas partes: segmentação (a) e fusão (b).

a) Segmentação Silábica: No caso da condição 'segmentação de palavras' apresentava-se à criança uma palavra e ela devia inserir o 'Pê' no começo de cada sílaba. A instrução era:

"Eu vou falar uma palavra e depois eu vou falar esta mesma palavra na "Língua do Pê". Para isto eu coloco o "Pê" na frente de cada pedaço da palavra. Veja como fica a palavra BOLA: fica PEBO - PELA. Vou falar agora outra palavra: CARRO. Na língua do "Pê" a palavra CARRO fica: PECA-PERRO, entendeu?

Agora é sua vez. Eu digo uma palavra e você a repete na "Língua do Pê". Vamos começar?"

Relação das palavras utilizadas na seção de teste: mesa, rato, fogo, belo, roda, magro.

No caso da condição 'segmentação de pseudopalavras', a seguinte instrução foi dada:

"Agora eu vou the dizer algumas palavras que não significam nada e quero que você faça como antes; ou seja, fale na "Língua do Pê".

Relação das pseudopalavras utilizadas na seção de teste: suni, cufa, meque, masa, pumi, tule.

b) Fusão Silábica: No caso da condição 'Fusão de palavras' apresentavase à criança palavras na "Língua do Pê", isto é, foi inserido no começo de cada sílaba das palavras enunciadas o "Pê". A instrução era:

“Agora vamos fazer o contrário. Eu falo uma palavra na "Língua do Pê" e você vai descobrir que palavra é esta. Por exemplo: 'PEDOPECE'. Neste exemplo, a palavra é "doce". Vou falar agora outra palavra: 'PESA-PEPO'.

Agora é você. Eu falo uma palavra na "Língua do Pê" e você diz que palavra é esta. Que palavra é 'pepo-pevo'?" 
Relação das palavras utilizadas na seção de teste: petu-pebo, peco-pelo, peso-pela, pete-pelha, peso-pegro, pema-peta.

No caso da condição 'fusão de pseudopalavras', a seguinte instrução foi dada:

"Agora eu vou lhe dizer algumas palavras sem sentido e quero que você faça como antes."

Relação das pseudopalavras utilizadas na seção de teste: pesa-pelho, pevu-pete, pefe-peta, pele-pepo, pero-pete, pefe-peba.

\section{Leitura}

- Tarefa Experimental de Leitura: Esta tarefa consiste na leitura em voz alta de um total de 28 estímulos, sendo 14 palavras e 14 pseudopalavras. Os estímulos variavam quanto ao número de sílabas (monossílabo ou polissílabo) e o grau de dificuldade (CV - V - CVC).

\subsection{Procedimentos}

As crianças foram avaliadas duas vezes. A primeira avaliação (Tempo 1) ocorreu quando as crianças estavam frequentando o último período da pré-alfabetização (outubro-novembro). Nesta primeira avaliação foram aplicados o subteste de Vocabulário do WISC, as Matrizes Progressivas de Raven - forma especial (Raven, 1987) e as duas tarefas que avaliam as habilidades de fusão e de segmentação: tarefa de fusão e segmentação fonêmica e tarefa de fusão e segmentação silábica. Dez meses depois, no final da alfabetização (setembro/outubro do ano seguinte), estas mesmas crianças foram também avaliadas pela segunda vez nas duas tarefas de consciência fonológica e em uma tarefa de leitura de palavras (Tempo 2 ).

\section{Resultados}

Devido à mudança de escola e desistência de participar da pesquisa, 19 crianças deixaram de realizar o teste de leitura de palavras no final do primeiro ano de alfabetização. A Tabela 2 apresenta as estatísticas descritivas das tarefas aplicadas.

Tabela 2 - Estatísticas Descritivas das Tarefas de Consciência Fonológica, I dade e Inteligência

\begin{tabular}{ccccccc}
\hline Variável & Esc. & Esc. & Média & DP & Ass. & Curt. \\
\hline Idade (meses) & 60 & 72 & 66,72 & 3,17 & 0,03 & $-0,92$
\end{tabular}


Antonio Roazzi, Maira M. Roazzi, Cláudia Nascimento Guaraldo Justi, Francis Ricardo dos Reis J usti A relação entre a habilidade de leitura e a consciência fonológica: estudo longitudinal em crianças pré-escolares

\begin{tabular}{lcccccc} 
Raven (esc. bruto) & 7 & 26 & 16,16 & 3,66 & $-0,17$ & 0,71 \\
WISC (esc. bruto) & 2 & 32 & 19,48 & 5,73 & $-1,02$ & 2,11 \\
Leitura (T2) & 1 & 6 & 4,50 & 1,74 & $-0,89$ & $-0,58$ \\
T1 Fusão Silábica & 0 & 12 & 1,00 & 2,48 & 3,34 & 11,28 \\
T1 Seg. Silábica & 0 & 12 & 0,71 & 2,46 & 3,74 & 13,68 \\
T1 Fusão Fonêmica & 0 & 9 & 0,82 & 1,68 & 2,87 & 9,38 \\
T1 Seg. Fonêmica & 0 & 5 & 0,13 & 0,65 & 6,72 & 49,52 \\
T2 Fusão Silábica & 0 & 11 & 2,04 & 3,58 & 1,60 & 1,03 \\
T2 Seg. Silábica & 0 & 12 & 2,60 & 4,52 & 1,35 & 0,02 \\
T2 Fusão Fonêmica & 0 & 8 & 1,26 & 2,52 & 1,86 & 2,08 \\
T2 Seg. Fonêmica & 0 & 10 & 0,74 & 2,34 & 3,82 & 13,82 \\
\hline
\end{tabular}

Nota. Esc = escore; Mín = mínimo; Máx = máximo; $D P=$ desvio padrão; Ass = assimetria; Curt = curtose; $\mathrm{T} 1=$ Tempo $1 ; \mathrm{T} 2=$ Tempo 2; Seg = segmentação.

Pode-se observar que houve variação considerável no desempenho nas tarefas de consciência fonológica, uma vez que, tanto tiveram crianças que não acertaram nenhum item quanto tiveram crianças que acertaram todos os itens (o escore máximo possível era 12). No entanto, de uma forma geral, as médias de acertos indicam que as tarefas foram bastante difíceis para as crianças. Considerando-se que o escore na variável dependente de interesse, a leitura, pode ser considerado dentro dos parâmetros de normalidade (assimetria e curtose entre 1 e -1), os dados foram analisados por meio de Análises de Regressão Linear Múltipla com passos fixos. No primeiro passo sempre foram incluídas juntas as variáveis de controle: idade, inteligência verbal (escore no WISC) e inteligência não verbal (escore no RAVEN). No segundo passo sempre se incluiu uma das tarefas de consciência fonológica. A Tabela 3 apresenta os resultados das análises de regressão considerando-se as medidas de consciência fonológica obtidas no último período da préalfabetização (tempo 1) e no final do primeiro ano de alfabetização (tempo 2).

Tabela 3 - Análises de Regressão Linear Múltipla Tendo Como Variável Dependente a Leitura e Como Variáveis Explicativas as Tarefas de Consciência Fonológica, a Idade, a Inteligência Verbal e Não Verbal

\begin{tabular}{clccc}
\hline Passo & \multicolumn{1}{c}{ Medida(s) } & Mud. em R & Mud. em F & Mud. em Sig. \\
\hline & & Tempo & & \\
1 & Idade, Int. V, Int. NV & 0,144 & 2,526 & 0,069 \\
2 & Fusão Silábica & 0,061 & 3,376 & 0,073 \\
2 & Seg. Silábica & 0,029 & 1,519 & 0,224 \\
2 & Fusão Fonêmica & 0,064 & 3,486 & 0,069
\end{tabular}


Antonio Roazzi, Maira M. Roazzi, Cláudia Nascimento Guaraldo Justi, Francis Ricardo dos Reis J usti A relação entre a habilidade de leitura e a consciência fonológica: estudo longitudinal em crianças pré-escolares

$\begin{array}{lllll}2 & \text { Seg. Fonêmica } & 0,024 & 1,232 & 0,273 \\ 1 & \text { Idade, Int. V, Int. NV } & 0,144 & 2,526 & 0,069 \\ 2 & \text { Fusão Silábica } & 0,085 & 4,861 & 0,033^{*} \\ 2 & \text { Seg. Silábica } & 0,032 & 1,702 & 0,199 \\ 2 & \text { Fusão Fonêmica } & 0,005 & 0,157 & 0,695 \\ 2 & \text { Seg. Fonêmica } & 0,001 & 0,018 & 0,894\end{array}$

Nota. Mud = mudança; Int = inteligência; $\mathrm{V}=$ verbal; $\mathrm{NV}=$ não verbal; Seg $=$ segmentação; $*=p<0,05$.

Como pode ser observado na Tabela 3 as variáveis 'idade', 'inteligência não verbal' (escore no RAVEN) e 'inteligência verbal' (escore no WISC), quando tomadas em conjunto, explicam aproximadamente $14 \%$ da variância na leitura. Porém a contribuição dessas variáveis controle para a leitura não chegou a ser significativa $(p=0,069)$. Considerando-se as tarefas de consciência fonológica realizadas no tempo 1 , pode-se dizer que, ao entrarem no segundo passo, as tarefas de fusão silábica e fonêmica, sozinhas, explicaram aproximadamente $6 \%$ da variação na habilidade de leitura no tempo 2. No entanto, a contribuição dessas variáveis também não chegou a ser estatisticamente significativa, embora tenha chegado perto disso $(p=0,073$ e $p=0,069$, respectivamente). Já as tarefas que envolveram segmentação silábica e fonêmica contribuíram com menos do que 3\% da variação na leitura quando entraram no segundo passo, não sendo essa uma contribuição estatisticamente significativa (todos os valores $p>0,20$ ).

Considerando-se os resultados das análises de regressão tendo como variáveis explicativas os escores nas tarefas de consciência fonológica realizadas no final do primeiro ano de alfabetização (tempo 2), pode-se observar que a tarefa de fusão silábica passa a explicar sozinha e de forma significativa $8,5 \%$ da variação na habilidade de leitura. Já as demais tarefas de consciência fonológica contribuem pouco e de forma não significativa ao entrarem no segundo passo da equação regressiva. Como as contribuições para a leitura da fusão silábica e fonêmica quando medidas no tempo 1 quase foram estatisticamente significativas decidimos explorar melhor a relação dessas variáveis com a leitura. Para tanto, como os participantes apresentaram um escore médio muito baixo nas tarefas de consciência fonológica, optamos por dicotomizar o escore nessas tarefas em 'participantes que não acertaram nenhum item' e 'participantes que acertaram, pelo menos, um item'. Os participantes foram divididos em dois grupos de acordo com os escores nas tarefas de consciência fonológica administradas no tempo 1 e utilizou-se o teste de Mann-Whitney U para comparar os escores desses 
grupos na habilidade de leitura medida no final do primeiro ano de alfabetização (tempo 2). Os resultados dos testes de Mann-Whitney U revelaram que as crianças que acertaram, pelo menos, um item na tarefa de fusão silábica tiveram um desempenho na leitura significativamente melhor do que as que não acertaram nenhum item (U $=174, z=-1,96, p=0,05)$. O mesmo ocorreu para as crianças que acertaram, pelo menos, um item na tarefa de fusão fonêmica em relação às que não acertaram nenhum item $(U=137, z=-2,977, p=$ 0,003 ). Porém, no que diz respeito às tarefas de segmentação silábica e fonêmica, as comparações entre as crianças que acertaram, pelo menos, um item e as que não acertaram, não foram estatisticamente significativas.

\section{Discussão}

Um dos objetivos desse estudo foi trazer evidências longitudinais que permitissem inferir a direção da relação entre a consciência fonológica e a leitura utilizando diferentes medidas de consciência fonológica. Se considerarmos os achados das análises de regressão, pode-se dizer que, à primeira vista, esses corroboram a proposta de Morais (1996) e são compatíveis com os achados de Mota e Castro (2007). Afinal, após um ano de escolarização a contribuição da tarefa de fusão silábica para a leitura aumentou de $6 \%$ para $8,5 \%$ e passou a contribuir significativamente para a leitura apenas quando medida no final do primeiro ano de alfabetização. Por outro lado, se considerarmos que as tarefas foram difíceis para os participantes (restringindo, dessa fora, a variação nos escores) e que devido a isso e ao número de participantes que deixou o estudo, talvez, as análises de regressão tenham perdido poder estatístico, torna-se relevante considerar, também, os resultados das análises não paramétricas. Nesse caso, a situação muda de figura, posto que os resultados dos testes de Mann-Whitney indicaram que ambas as tarefas de fusão (silábica e fonêmica) medidas antes da alfabetização predisseram de forma estatisticamente significativa o desempenho em leitura um ano depois. É interessante considerar que o mesmo não ocorreu para as tarefas de segmentação e que, além disso, nas análises prévias de regressão essas tarefas de fusão ficaram próximas do critério de significância estatística. Esse padrão se coaduna perfeitamente com a hipótese de que pode ter faltado poder estatístico na análise de regressão. Assim sendo, a interpretação da relação entre a consciência fonológica e a leitura que melhor corresponde aos achados desse estudo é a da causação recíproca, porque se por um lado pode-se argumentar que as tarefas de fusão silábica e fonêmica no tempo 1 
predisseram o desempenho em leitura no tempo 2, por outro, a tarefa de fusão silábica, medida no final do primeiro ano de alfabetização, se relacionou mais fortemente com a leitura.

Outra questão que esse estudo objetivou abordar foi a possível diferença entre as tarefas que envolvem fusão e segmentação de unidades fonológicas em sua relação com a leitura. Nesse sentido, tanto nas análises de regressão quanto nas análises não paramétricas pôde-se observar de forma clara que as tarefas que envolviam fusão (principalmente a fusão silábica) se relacionaram mais fortemente com a leitura do que as tarefas que envolviam segmentação. Esse dado de que as tarefas que envolvem fusão se relacionam mais fortemente com a leitura replica, em parte, os achados de Perin (1983) e Fox e Routh (1984). No entanto, qualquer afirmação conclusiva nessa direção seria precipitada, afinal, as tarefas de segmentação foram mais difíceis do que as tarefas de fusão e isso pode ter restringido ainda mais a variação dos escores nas primeiras, dificultando assim a detecção de uma possível relação dessas com a leitura. Cabe lembrar que no estudo de Cardoso-Martins (1995b) realizado com pré-escolares brasileiros, tanto a tarefa de segmentação fonêmica quanto a tarefa de detecção silábica predisseram a leitura. Porém, cabe lembrar também que, nesse mesmo estudo de Cardoso-Martins, a tarefa de segmentação fonêmica também foi a mais difícil (as crianças acertaram em média 2,65 itens de um total de 15). Assim sendo, a questão que talvez se deva colocar aqui é: por que as tarefas de que envolvem segmentação são mais difíceis do que as que envolvem fusão?

Os dados do presente estudo não têm como responder diretamente à questão posta. No entanto, pode-se, pelo menos, hipotetizar a esse respeito. Uma possibilidade é que como para responder nas tarefas de segmentação empregadas nesse estudo, as crianças tinham que produzir respostas com mais unidades (p.ex.: para a palavra 'bola' a criança tinha que produzir duas unidades ao segmentar, 'pebo' e 'pela', porém ao aglutinar ela deveria produzir só uma, 'bola'), torna-se proporcionalmente maior a chance de erro na geração da resposta. Além disso, se considerarmos que devido à idade das crianças essas tarefas implicavam em fortes demandas à memória de trabalho das crianças e que devido à dificuldade das tarefas, as crianças poderiam realizá-las de forma lenta, quanto mais tempo elas gastassem gerando uma unidade de resposta, maior seria a chance da informação original não ser mantida na memória de trabalho, seja por interferência ou pela mera passagem do tempo. Afinal, crianças pré-escolares não costumam utilizar estratégias espontaneamente para manter uma informação na memória de trabalho (FLAVELL; MILLER; MILLER, 1999). Assim sendo, pode ser que a diferença entre esses dois tipos de tarefa (fusão e 
segmentação) se deva a uma capacidade ainda muito limitada de memória de trabalho dos pré-escolares e que em crianças mais velhas essa diferença desapareça. Estudos futuros poderiam explorar essa possibilidade ao investigar se a diferença de desempenho nas tarefas que envolvem fusão e segmentação fonêmica e silábica é mediada pela capacidade de memória de trabalho dos participantes.

Ainda com relação às tarefas utilizadas, pode-se dizer que, de forma consistente, as crianças obtiveram menores escores nas tarefas que envolviam fonemas enquanto unidades do que nas tarefas que envolviam sílabas. Esta maior dificuldade da tarefa fonêmica em relação à tarefa silábica não é novidade (e.g., CHOMSKY, 1977; EHRI, 1979; LIBERMAN, 1977), e mostra como a segmentação fonêmica é a habilidade de segmentação mais difícil e cuja aquisição por parte da criança é um forte indicador de um nível de consciência linguística adiantado e mais sofisticado. A sílaba por ser uma unidade de articulação básica, possui uma maior saliência perceptual e é, portanto, também mais facilmente identificada e produzida de forma consciente. Já, o fonema não é um índice fácil de ser definido, nem invariante a nível acústico (GIBSON, 1972). Os fonemas, de fato, como sugerem análises espectrográficas, se articulam entre eles; nas palavras, cada fonema engloba um pouco de informação do fonema anterior e daquele que o segue (TUNMER; PRATT; HERRIMAN, 1984). Os fonemas são mais uma abstração convencional, que se tem imposto funcionalmente durante o processo de desenvolvimento histórico da língua escrita por causa de sua maior conveniência em relação a uma escrita de tipo silábico. Implica a utilização de um número menor de signos (GELB, 1972). De fato, a segmentação natural seria sem dúvida a silábica, dado que para esta existe um preciso e definido correspondente a nível acústico (ZUCCHERMAGLIO, 1985).

Por fim, é importante pontuar algumas limitações do presente estudo. Independente de se ter conseguido detectar uma diferença entre as tarefas que envolviam fusão e as que envolviam segmentação, é importante considerar que o baixo nível de desempenho das crianças nessas tarefas de uma forma geral, levanta dúvidas quanto à adequação dessas tarefas para avaliar a consciência fonológica de pré-escolares. Seria importante que estudos futuros tentassem desenvolver versões mais simples dessas tarefas, mas que, ainda assim, permitissem investigar as questões em tela. Trata-se de uma preocupação necessária, principalmente, se considerarmos que, em estudos longitudinais é muito comum a perda de participantes, o que também pode contribuir para diminuir a variabilidade dos escores. Nesse caso, não se pode deixar de considerar que a redução do número de participantes na segunda coleta e a baixa variabilidade dos escores de 
Antonio Roazzi, Maira M. Roazzi, Cláudia Nascimento Guaraldo J usti, Francis Ricardo dos Reis J usti A relação entre a habilidade de leitura e a consciência fonológica: estudo longitudinal em crianças pré-escolares

consciência fonológica podem ter contribuído para reduzir o poder estatístico das análises de regressão empregadas no presente estudo.

\section{Referências}

BALL, E.; BLACHMAN, B. Does phoneme awareness training in kindergarten make a difference in early word recognition and development spelling? Reading Research Quarterly, Hoboken, New Jersey, v. 26, p. 49-66, 1991.

BARRERA, L. Consciência metalinguística e alfabetização: um estudo com crianças da primeira série do ensino fundamental. Psicologia: Reflexão e Crítica, Porto Alegre, v.16, p.491-502, 2003.

BERNARDINO JÚNIOR, J. A.; FREITAS, F.; SOUZA, D.; MARANHE, E.; BANDINI, H. Aquisição de leitura e escrita como resultado do ensino de habilidades de consciência Fonológica. Revista Brasileira de Educação Especial, Marilia, v. 12, n. 3, p. 423-450, 2006.

BERTELSON, P.; DE GELDER, B. The emergence of phonological awareness. Em I. Mattingly \& M. Studdert-Kennedy (Eds.), The motor theory of speech perception. Hillsdale, N.J.: Lawrence Erlbaum Associates, 1991.

BOWEY, J. Phonological sensitivity in novice readers and nonreaders. J ournal of Experimental Child Psychology, Amsterdam, v. 58, n. 1, 134-159, 1994.

BRADLEY, L.; BRYANT, P. Categorizing sounds and learning to read: a causal connection. Nature, London, v. 301, n. 1, 419-421, 1983.

BRADY, S.; FOWLER, A., STONE, B.; WINBURY, N. Training phonological awareness: a study with inner-city kindergarten children. Annals of Dyslexia, New York, v. 44, n. 1, 26-59, 1994.

BRYANT, P. Phonological awareness is a pre-cursor, not a pre-requisite, of reading. Mind \& Language, Hoboken, New Jersey, v. 6, n. 2, 102106, 1991.

BRYANT, P.; BRADLEY, L. Children's reading problems. Oxford: Blackwell, 1985.

BRYANT, P.; MACLEAN, M., BRADLEY, L.; CROSSLAND, J. Rhyme and alliteration, phoneme detection, and learning to read. Developmental Psychology, Washington, v. 26, n. 1, p. 429-438, 1990.

BURGESS, S.; LONIGAN, C. Bidirectional relations of phonological sensitivity and prereading abilities: evidence from a preschool sample. Journal of Experimental Child Psychology, Amsterdam, v. 70, n .1 117-141, 1998.

BYRNE, B.; FIELDING-BARNSLEY, R. Evaluation of a program to teach phonemic awareness to young children: a 2- and 3- year follow-up and 
Antonio Roazzi, Maira M. Roazzi, Cláudia Nascimento Guaraldo J usti, Francis Ricardo dos Reis J usti A relação entre a habilidade de leitura e a consciência fonológica: estudo longitudinal em crianças pré-escolares

a new preschool trial. Journal of Educational Psychology, Washington, v. 87, n. 1, p. 488-503, 1995.

CAPOVILLA, A.; CAPOVILLA, F. Efeitos do treino de consciência fonológica em crianças com baixo nível sócio-econômico. Psicologia: Reflexão e Crítica, Porto Alegre, v. 13, n. 1,7-24, 2000.

CARDOSO-MARTINS, C. A consciência fonológica e a aprendizagem inicial da leitura e da escrita. Cadernos de Pesquisa, São Paulo, v. 5, n. 1, p. 41-49, 1991.

CARDOSO-MARTINS, C. Rhyme perception: global or analytical? J ournal of Experimental Child Psychology, Amsterdam, v. 57, n. 1, p. 26-41, 1994.

CARDOSO-MARTINS, C. Sensitivity to rhymes, syllables and phonemes in literacy acquisition in Portuguese. Reading Research Quarterly, Hoboken, New Jersey, v. 30, n. 1, p. 808-828, 1995b.

CARDOSO-MARTINS, C. Consciência fonológica e alfabetização. Petrópolis: Vozes, 1995a.

CÁRNIO, M.; SANTOS, D. Evolução da consciência fonológica em alunos de ensino fundamental. Pró-Fono: Revista de Atualização Científica, Barueri, v. 17, n. 2, p. 195-200, 2005.

CASTLES, A.; COLTHEART, M. Is there a causal link from phonological awareness to success in learning to read. Cognition, Amsterdam, v. 91, n. 1, p. 77-111, 2004.

CASTLES, A.; HOLMES, V.; NEATH, J.; KINOSHITA, S. How does orthographic knowledge influence performance on phonological awareness tasks? Quarterly Journal of Experimental Psychology, Washington, v. 56, n. 1, p. 445-467, 2003.

CHOMSKY, N. Approaching reading through invented spelling. Em L.B. Resnick \& P.A. Weaver (Eds.), Theory and practice of early reading. Vol. 2. Hillsdale, N.J .: Lawrence Erlbaum Associates, 1977.

COSSU, G.; SHANKWEILER, D.P. LIBERMAN, I.Y. KATZ, L.; TOLA, G. Awareness of phonological segments and reading ability in Italian children. Applied Psycholinguistics,Cambridge, v. 9, n. 1, p. 1-16, 1988.

DE GELDER, B. Phonological awareness, misidentification and multiple identities. In: J. A. Edmondson, C. Feagin \& P. Mülhauser (Eds.), Development and diversity: linguistic variation across time and space. Arlington: Texas Publications, 1990.

DE GELDER, B.; VROOMEN, J. Phonological deficits: Beneath the surface of reading-acquisition problems. Psychological Research, New York, v. 53, n. $1,88-97,1991$. 
DILLON, C.; JONG, K.; PISONI, D. Phonological Awareness, Reading Skills, and Vocabulary Knowledge in Children Who Use Cochlear Implants, Journal of Deaf Studies and Deaf Education, doi: 10.1093/deafed/enr043. Published online: November 3, 2011 <http://jdsde.oxfordjournals.org/content/early/2011/11/03/deafed.enr0 43. short?rss $=1>$

EHRI, L. C. Linguistic insight: threshold of reading acquisition. Em T.G. Waller \& G.E. Mc Kinnon (Eds.), Reading research advances: theory and practice. Vol. 1. New York: Academic Press, 1979.

FLAVELL, J.; MILLER, P.; MILLER, S. Desenvolvimento cognitivo. Porto Alegre: ArtMed, 1999. FOX, B.; ROUTH, D. Phonemic analysis and synthesis as word attack skills: Revisited. Journal of Educational Psychology, Washington, v. 76, n. 1, p. 1059-1064, 1984.

FUKUDA, M.; CAPELLINI, S. Programa de Intervenção Fonológica Associado à Correspondência Grafema-Fonema em Escolares de Risco para a Dislexia. Psicologia: Reflexão e Crítica, Porto Alegre, v. 25, n. 4, p. 783-790, 2012.

GIBSON, E. J. Reading for some purpose. In: J.F. Kavanagh \& I.G. Mattingly (Eds.), Language by ear and by eye: the relationship between speech and reading. Cambridge Mass.: MIT Press, 1972.

GOSWAMI, U.; BRYANT, P. Phonological skills and learning to read. Hillsdale, NJ: Erbaum, 1990.

GOUGH, P.; LARSON, K.; YOPP, H. A estrutura da consciência fonológica. In C. Cardoso-Martins (Ed.), Consciência fonológica e alfabetização. Petrópolis: Vozes, 1995, p. 15-35.

HEIN, J.; TEIXEIRA, M.; SEABRA, A.; MACEDO, E. Avaliação da eficácia do software "alfabetização fônica" para alunos com deficiência mental. Revista Brasileira de Educação Especial, Marilia, v. 16, n.1, p. 6582, 2010.

INVERIZZI, M. Concepts, sounds, and the ABCs: A diet for the very young reader. In: D. Barone \& L. M. Morrow (Eds.) . Literacy and young children: Research-based practices. New York: Guilford, 2003, p. 140- 156.

JORM, A.F.; SHARE, D.L. Phonological recoding and reading acquisition. Applied Psycholinguistics, Cambridge, v. 4, n. 1, p. 103-147, 1983. JUSTINO, M.; BARRERA, S. Efeitos de uma Intervenção na Abordagem Fônica em Alunos com Dificuldades de Alfabetização. Psicologia: Teoria e Pesquisa, Brasília, v. 28, n. 4, p. 399-407, 2012.

LIBERMAN, I. Y. Phonetic segmentation and recording in the beginning reader. In: R. S. Reber \& D.L. Scarborough (Eds.), Towards a psychology of reading: the Proceedings of the CUNY Conference. New York: Wiley, 1977. 
LUNDBERG, I.; HOIEN, T. Initial enabling knowledge and skills in reading acquisition: Print awareness and phonological segmentation. In: D. Z. J. Sawyer \& B. J. Fox (Eds.), Phonological awareness in reading: the evolution of current perspectives. Berlin: Springer, 1991. MALUF, R.; BARRERA, S. Consciência fonológica e linguagem escrita em pré-escolares. Psicologia: Reflexão e Crítica, Porto Alegre, v. 10, n. 1, p. 125-145, 1997.

MANRIQUE, A.; DE SIGNORINI, A. Del habla a la escritura: la consciência linguística como una forma de transición natural. Lectura y Vida, Buenos Aires, v. 9, n. 2, p. 5-9, 1988.

MORAIS, J.; ALEGRIA, J.; CONTENT, A. The relationships between segmental analysis and alphabetic literacy: An interactive view. Cahiers de Psychologie Cognitive, Paris, v. 7, n. 1, p. 415-438, 1987.

MORAIS, J. A arte de ler. São Paulo: Editora da Universidade Estadual Paulista, 1996.

MOTA, M. Introdução: desenvolvimento metalinguístico. In M. Mota (Ed.), Desenvolvimento metalinguístico: questões contemporâneas. São Paulo: Casa do Psicólogo, 2009, p. 10-18.

MOTA, M.; CASTRO, N. Alfabetização e consciência metalinguística: um estudo com adultos não alfabetizados. Estudos de Psicologia, Campinas, v. 24, n. 2, p. 169-179, 2007.

OLOFSSON, A.; LUNDBERG, I. Evaluation of long term effects of phonemic awareness training in kindergarten. Scandinavian Journal of Psychology, Stockholm, v. 26, n. 1, 21-34, 1985.

PAULA, J.; MOTA, H.; KESKE-SOARES, M. A terapia em consciência fonológica no processo de alfabetização. Pró-Fono Revista de Atualização Científica, Barueri, v. 17, n. 2, p. 175-184, 2005.

PERFETTI, C. A.; BECK, I.; BELL, L.; HUGHES, C. Phonemic knowledge and learning to read are reciprocal: A longitudinal study of first grade children. Merrill-Palmer Quarterly, Detroit, v. 33, n. 1, p. 283-320, 1987.

PERIN, D. Phonemic segmentation and spelling. British Journal of Psychology, Hoboken, New Jersey, v. 74, n. 1, p. 129-144, 1983.

PESTUN, M. Consciência fonológica no início da escolarização e o desempenho ulterior em leitura e escrita: estudo correlacional. Estudos de Psicologia, Natal, v. 10, n. 3, 407-412, 2005.

PHILLIPS, B.; TORGESEN, J. Phonemic awareness and reading: Beyond the growth of initial reading accuracy. Handbook of early literacy research . Vol. 2, New York: Guilford, 2006, p. 101-113.

RAVEN, J. C. Matrizes progressivas de Raven - Séries A, Ab, B Escala especial. São Paulo: Casa do Psicólogo, 1987. 
Antonio Roazzi, Maira M. Roazzi, Cláudia Nascimento Guaraldo Justi, Francis Ricardo dos Reis J usti A relação entre a habilidade de leitura e a consciência fonológica: estudo longitudinal em crianças pré-escolares

ROAZZI, A. Metalinguistic awareness in Brazilian bards. Trabalho apresentado no 40 Congrès Internacional - 'Association pour la Recherche Interculturelle' e XIO International Congress 'International Association for Cross-Cultural Psychology' Université de Liège, 15 à 18 J ulho de 1992.

ROAZZI, A. Como prever habilidades de leitura a partir do desenvolvimento da consciência fonológica: um estudo longitudinal em crianças de pré-escolar. Trabalho apresentado na XVI Reunião Anual da ANPED (Associação Nacional de Pós-Graduação e Pesquisa em Educação. GT de Alfabetização 'Alfabetização: Diferentes perspectivas Teórico-Metodológicas. Caxambú, 1993.

ROAZZI, A; ASFORA, R.; QUEIROGA, B.; DIAS, M. G. B. B. Competência metalinguística antes da escolarização formal. Educar em Revista, Curitiba, 38, Set/Dez, p. 43-56, 2010.

ROAZZI, A.; CARVALHO, M. R. O desenvolvimento de habilidades de segmentação lexical e a aquisição da leitura. Revista Brasileira de Estudos Pedagógicos, Brasilia, v. 76, n. 184, p. 477-548, 1995.

ROAZZI, A.; DOWKER, A. Consciência fonológica, rima e aprendizagem da leitura. Psicologia: Teoria e Pesquisa, Brasilia, v. 5 , n. 1, p. 3155, 1989.

ROAZZI, A.; DOWKER, A.; BRYANT, P. Phonological abilities of Brazilian street poets. Applied Psycholinguistic, Cambridge, v. 14, n. 4, p. 535-551, 1993.

SCHATSCHNEIDER, C.; FRANCIS, D.; FOORMAN, B.; FLETCHER, J. The dimensionality of phonological awareness: an application of item response theory. Journal of Educational Psychology, Washington, v. 91, n. 1, p. 439-449, 1999.

STANOVITCH, K. E.; CUNNIGHAM, A. E.; CRAMER, B. Assessing phonological skills in kindgarten children: Issues of task comparability. J ournal of Experimental Child Psychology, Amsterdam , v. 38, n. 1, p. 175-190, 1984.

STANOVITCH, K. E.; CUNNIGHAM, A. E.; FEEMAN, D. Intelligence, cognitive skills and early reading progress. Reading Research Quarterly, Hoboken, New Jersey, v. 19, n.1, 278-303, 1984.

SHARE, D. L.; JORM, A.R.; MACLEAN, R.; MATTEWS, R. Sources of individual differences in reading acquisition. Journal of Educational Psychology, Washington, v. 76, n. 6, p. 1309-1324, 1984.

SNOWLING, M. Dyslexia: a cognitive development perspective. Oxford: Blackwell, 1987.

TRESSOLDI, P. E. Lo sviluppo della lettura e della scrittura. Età Evolutiva, Firenze, v. 33, giugno, p. 53-58, 1989.

TUNMER, W.E.; PRATT, C.; HERRIMAN, M. Metalinguistic awareness in children. New York: Springer-Verlag, 1984. 
Antonio Roazzi, Maira M. Roazzi, Cláudia Nascimento Guaraldo Justi, Francis Ricardo dos Reis J usti A relação entre a habilidade de leitura e a consciência fonológica: estudo longitudinal em crianças pré-escolares

VLOEDGRAVEN, J.; VERHOEVEN, L. The nature of phonological awareness throughout the elementary grades: an item response theory perspective. Learning and I ndividual Differences, Amsterdam, v. 19, n. 2, p. 161-169, 2009.

WAGNER, R.; TORGESEN, J. The nature of phonological processing and its causal role in the acquisition of reading skills. Psychological Bulletin, Washington, v. 101, n. 2, p. 192-232, 1987.

WAGNER, R.; TORGESEN, J.; RASHOTTE, C. Development of readingrelated phonological processing abilities: new evidence of bidirectional causality from a latent variable longitudinal study. Developmental Psychology, Washington, v. 30, n. 1, p. 73-87, 1994.

WECHSLER, D. WISC-III: Escala de inteligência Wechsler para crianças. Manual/David Wechsler, $3^{a}$ ed.; Adaptação e padronização brasileira, $1^{a}$ ed.; Vera Lúcia Marques de Figueiredo. São Paulo: Casa do Psicólogo, 1991/2002.

WI MMER, H.; LANDERL, K.; LINORTNER, R.; HUMMER, P. The relationship of phonemic awareness to reading acquisition: more consequence than precondition but still important. Cognition, Amsterdam, v. 40, n. 1, p. 219-249, 1991.

ZUCCHERMAGLIO, C. II cammino del bambino verso l'appropriazione della lingua scritta. Etá Evolutiva, Firenze, v. 21, giugno, p. 95-104, 1985.

\section{Endereço para correspondência \\ Antonio Roazzi}

Universidade Federal de Pernambuco, $\mathrm{CFCH} 80$ andar, Av. Arquitetura s/n, Cidade Universitária, CEP 50740-550, Recife, PE - Brasil.

Endereço eletrônico: roazzi@gmail.com

Maira M. Roazzi

Instituto de Psicologia/UFRGS, Rua Ramiro Barcelos, 2600/104, CEP 90035-

003, Porto Alegre, RS - Brasil.

Endereço eletrônico: maira.roazzi@gmail.com

Cláudia Nascimento Guaraldo J usti

Universidade Federal de Juiz de Fora, Instituto de Ciências Humanas, Departamento de Psicologia, CEP 36036-900 - Juiz de Fora, MG - Brasil.

Endereço eletrônico: claudia.ngjusti@gmail.com

\section{Francis Ricardo dos Reis J usti}

Universidade Federal de Juiz de Fora, Instituto de Ciências Humanas, Departamento de Psicologia, CEP 36036-900 - Juiz de Fora, MG - Brasil.

Endereço eletrônico: francisjusti@gmail.com

Recebido em: 24/05/2012

Reformulado em: 04/06/2013

Aceito para publicação em: 06/06/2013

Acompanhamento do processo editorial: Adriana Benevides Soares 
Antonio Roazzi, Maira M. Roazzi, Cláudia Nascimento Guaraldo Justi, Francis Ricardo dos Reis J usti A relação entre a habilidade de leitura e a consciência fonológica: estudo longitudinal em crianças pré-escolares

\section{Notas}

* Doutor (D. Phil.) em Psicologia do Desenvolvimento pela University of Oxford obtido em 1988 sob a orientação do Prof. Peter E. Bryant (superviser) e Prof. Donald Broadbent (Adviser). Possui também titulo de "Dottore" em Psicologia Aplicada pela Universitá degli Studi di Roma "La Sapienza". Fez pós-doutorado na University of London, University of Oxford e Instituto Di Scienze e Tecnologie Della Cognizione, ISTC, Roma, Itália. Pesquisador Nivel 1A do CNPq, foi membro de Comitês de Avaliação do CAPES, ENEM e FACEPE. Membro Titular (2000-2002) e Coordenador do Comitê de Assessoramento de Psicologia e Serviço Social do CNPq (CA-PS, 20112012). Tem experiência na área de Psicologia, com ênfase em Psicologia do Desenvolvimento Sócio-Cognitivo. Ultimamente atua principalmente nos seguintes temas: consciência e autoconsciência, ayahuasca e estados ampliados de consciëncia, psicologia do desenvolvimento sócio-cognitivo, aprendizagem da leitura e escrita, lógica mental, teoria da mente, percepção de risco e apego. Foi Presidente da Sociedade Brasileira de Psicologia do Desenvolvimento (2002-2004) e da Facet Theory Association (2011-2013).

** Possui graduação em psicologia clinica pela Universidade Católica de Pernambuco (2004), mestrado em Psicologia Cognitiva pela Universidade Federal de Pernambuco (2007) e doutorado em Psicologia do Desenvolvimento pela University of Pittsburgh. Atualmente é pesquisadora de Pós-doutorado no Instituto de Psicologia da UFRGS. Tem experiência na área de Psicologia, com ênfase em Psicologia Cognitiva, Psicologia do Desenvolvimento, e Ciência Cognitiva da Religião (Cognitive Science of Religion), atuando principalmente nos seguintes temas: desenvolvimento de conceitos de morte e vida após a morte, raciocínio implícito (acerca de mente, alma, espirito, e energia vital), desenvolvimento de uma biologia ingênua (naive biology). Seu foco de pesquisa é transcultural já tendo realizado pesquisas no Brasil, EUA, Indonésia e China.

***Possui graduação em Psicologia pela Universidade Federal de Juiz de Fora (2000) e mestrado em Psicologia(área de concentração em Psicologia do Desenvolvimento Humano)pela Universidade Federal de Minas Gerais(2006). Doutorou-se em Psicologia Cognitiva pela Universidade Federal de Pernambuco(2009). Tem experiência na área de Psicologia, com ênfase em Psicologia do Desenvolvimento Humano, Psicologia Cognitiva e Psicologia Escolar. Atualmente é professora adjunta do curso de Psicologia da UFJF e tem desenvolvido pesquisas, principalmente, sobre os seguintes temas: processos cognitivos subjacentes ao desenvolvimento da leitura e da escrita e modelos computacionais de reconhecimento visual de palavras.

**** Possui graduação em Psicologia pela Universidade Federal de Juiz de Fora (2003) e mestrado em Psicologia (área de concentração em Psicologia do Desenvolvimento Humano) pela Universidade Federal de Minas Gerais (2006). Doutorou-se em Psicologia (área de concentração em Psicologia Cognitiva) pela Universidade Federal de Pernambuco. Tem experiência na área de Psicologia, com ênfase em Psicologia Cognitiva, atuando principalmente nos seguintes temas: reconhecimento visual de palavras, linguagem (oral e escrita) e processos psicológicos básicos de uma forma geral. 